\title{
Rationing of Care: Conceptual Ambiguity and Transparency in Data Collection and Synthesis
}

\author{
Rebekah J. Walker, $P h D^{1,2,3}$ and Leonard E. Egede, MD,MS \\ ${ }^{1}$ Health Equity and Rural Outreach Innovation Center (HEROIC), Ralph H. Johnson Veterans Affairs Medical Center, Charleston, SC, USA; ${ }^{2}$ Center for \\ Health Disparities Research, Medical University of South Carolina, Charleston, SC, USA; ${ }^{3}$ Department of Medicine, Division of General Internal \\ Medicine and Geriatrics, Medical University of South Carolina, Charleston, SC, USA.
}

J Gen Intern Med 31(12): 1415-6

DOI: $10.1007 / \mathrm{s} 11606-016-3801-4$

(๑) Society of General Internal Medicine 2016

$\mathrm{R}$ ationing continues to be a hotly debated and controversial issue, with great disagreement over not only whether rationing is acceptable, but what it means to ration care. In this issue of JGIM, Sheeler et al. present findings of a crosssectional study that aims to describe physician rationing behaviors and characteristics of rationing in the United States. ${ }^{1}$ Surveys mailed to physicians randomly selected from the American Medical Association master file asked about frequency with which they "personally refrained, because of cost to the health care system," from using ten different interventions, including laboratory tests, magnetic resonance imaging (MRI), referral, or prescription drugs. They found overall that $53 \%$ of respondents reported refraining from using services in the past 6 months. ${ }^{1}$ Prescription drugs and MRI were most frequently reported at $48 \%$ and $45 \%$, respectively. ${ }^{1}$ Surgical and procedural specialists, medical school settings, and physicians who self-identified as very or somewhat politically conservative reported higher frequency of refraining from using at least one of the ten services than primary care, small or solo practice, and those identifying as very or somewhat liberal. ${ }^{1}$ In addition, the survey asked respondents one of three randomly assigned questions to gauge perceived responsibility for containing costs, phrasing questions either as responsibility to "exercise wise financial resource stewardship," "promote cost consciousness" or "ration in my daily care of patients." Compared to $88 \%$ agreeing with the "wise-stewardship" and $81 \%$ agreeing with the "cost-conscious" statements, only $22 \%$ agreed with the "rationing" statement. ${ }^{1}$

As seen in the results reported by Sheeler et al., the phrasing used to ask about rationing has an impact on responses. As a result, it is necessary to be clear regarding the definition of rationing. Ubel and Goold suggested in 1998 the use of a broad interpretation "encompassing any explicit or implicit measures that allow people to go without beneficial health care services." ${ }^{2}$ They argued that this would highlight the frequency with which rationing occurs, and remove the de facto negative connotation. ${ }^{2}$ However, discussions of

Published online July 19, 2016 rationing over the past 5 years consistently note there is no agreed upon definition, and point out that the term is often used synonymously with ideas such as 'resource allocation,' 'priority setting' and 'cost containment. ${ }^{3-6}$ The political processes surrounding the Affordable Care Act in the United States, and the National Health Service in the United Kingdom have added to the conceptual and methodological struggle to measure and understand clinician attitudes and willingness to accept rationing either in their own practice or through policy measures. ${ }^{3-5}$ Even what is considered rationing is unclear; for example, limitations on services by ability to pay, or lack of care resulting from lack of health insurance, is seen as implicit rationing by some definitions, but not others. ${ }^{2},{ }^{5},{ }^{6}$ By not directly asking about rationing, Sheeler et al. avoided possible reactions to the term itself, and stated this could prompt more professional discourse on the topic. ${ }^{1}$ However, given the widespread disagreement on what does and does not constitute rationing, the conclusions drawn could be inadvertently misleading and may be inconsistent with the intent of survey participants at the time they answered the questions.

Previous studies have investigated the willingness of physicians to ration health care as well as their perceptions of rationing. Hurst et al. used a similarly phrased questionnaire in Norway, Switzerland, Italy, and the United Kingdom, and found a similar overall response, with $56 \%$ of physicians refraining from at least one of the ten interventions in the past 6 months. ${ }^{7}$ When asked more detailed questions regarding criteria for rationing, respondents most often reported a small expected benefit $(82 \%)$ and low chance of success $(80 \%){ }^{7} \mathrm{~A}$ national survey of intensive care units (ICU) in the United States found that a majority of respondents perceived questions about specific situations or practices that may be associated with rationing occurred "rarely" or "not at all." ${ }^{8}$ Few ICUs perceived rationing as occurring "frequently"; however, $46 \%$ of respondents felt "too much care" was provided "sometimes or frequently." In this study, the word rationing was used, but not until the end of the survey in an effort to minimize bias resulting from varied definitions around the concept of rationing. ${ }^{8}$ Interestingly, a high prevalence of perceived rationing was reported when a similar survey of the Society of Critical Care Medicine membership asked about perceived rationing by other practitioners, rather than by the respondents. ${ }^{8}$ More recently, a systematic review of survey 
research concerning physician attitudes towards rationing health care found conflicting results, with anywhere from $9 \%$ to $94 \%$ of respondents willing to accept rationing. ${ }^{9}$ The review defined rationing as withholding beneficial interventions for cost reasons, and found significant differences in question wording, which they felt was partially due to the lack of a universally accepted definition or clear conceptual basis for questions. ${ }^{9}$ Many studies avoided the term rationing, instead focusing on considerations of cost or cost-effectiveness in patient care; however, authors note that since cost considerations do not necessarily include rationing, this may be misleading. ${ }^{9}$ Seven studies asked about different rationing strategies, but most studies did not provide details on why or how costs should be contained. ${ }^{9}$

Valid, empirical evidence is needed surrounding physician approaches and attitudes towards rationing; however, variable and contentious views regarding the definition and scope of rationing has hampered comprehensive findings. ${ }^{9}$ The findings of Sheeler et al. add to the literature in that they provide an estimate of United States physicians reporting rationing, without focusing on one specific sub-specialty. The study also provides information on attitudes towards resource allocation, and highlights the everyday clinical decisions physicians make taking cost, risks and benefits into account. ${ }^{1}$ However, major limitations exist in the way questions were asked and the behavioral definition used to frame questions without directly asking about rationing. As a result, the findings should be interpreted with caution when reporting rationing behavior and the differences between groups. The systematic review conducted by Strech et al. noted that willingness to accept rationing decreases when the aims or consequences of decisions are included in the question. ${ }^{9}$ Therefore, estimates may vary significantly when consequences are specified or when specific words like "rationing" or "denying" care are used. Future research should use precise wording that avoids conceptual ambiguity. ${ }^{9}$ While many authors support avoiding the term rationing, it is imperative that questions have a solid theoretical basis and should address factors that influence decisions to ration care and preferences for implicit as opposed to explicit strategies. ${ }^{9}$ If clinicians are to be meaningfully involved in the ongoing discussion on rationing care through their responses to surveys, it is critical that such surveys - as well as the policies and guidelines that follow-be conceptually coherent. In this important area of study, clarity and transparency should never be rationed.

Corresponding Author: Leonard E. Egede, MD,MS; Center for Health Disparities ResearchMedical University of South Carolina, Charleston, SC, USA (e-mail: egedel@musc.edu).

\section{Compliance with Ethical Standards:}

Conflict of Interest: The authors have no financial disclosure or conflict of interest to report.

\section{REFERENCES}

1. Sheeler RD, Mundell T, Hurst SA, Goold SD, Humeniuk KM, Thorsteinsdottir B, et al. Self-reported rationing behavior among US physicians: a national survey. JGIM. 2016. doi:10.1007/s11606-0163756-5.

2. Ubel P, Goold S. Rationing health care: not all definitions are created equal. Arch Intern Med. 1998;158:209-214.

3. Klein R. Rationing in the fiscal ice age. Health Econ Policy Law. 2010;5:389396.

4. Callahan D. Rationing: theory, politics, and passions. Hastings Cent Rep. 2011;41(2):23-27.

5. Cohen A. The debate over health care rationing: Déjà vu all over again? Inquiry. 2012;49:90-100.

6. Scheunemann $\mathbf{L}$, White $\mathbf{D}$. The ethics and reality of rationing in medicine. Chest. 2011;140(6): 1625-1632.

7. Hurst S, Slowther AM, Forde R, Pegoraro R, Reiter-Theil S, Perrier A, et al. Prevalence and determinants of physician bedside rationing. JGIM. 2006;21:1138-1143.

8. Ward N, Teno J, Curtis R, Rubenfeld G, Levy M. Perceptions of cost constraints, resource limitations, and rationing in the United States intensive care units: results of a national survey. Crit Care Med. 2008;36(2):471-476.

9. Strech D, Persad G, Marckmann G, Danis M. Are physicians willing to ration health care? Conflicting findings in a systematic review of survey research. Health Policy. 2009;90:113-124. 